\title{
Magnetic Fields and Large-Scale SXR and EUV Coronal Structures
}

\author{
Lidia van Driel-Gesztelyi ${ }^{1,2,3}$ \\ ${ }^{1}$ Mullard Space Science Laboratory, University College London, UK \\ email: lvdg@mssl.ucl.ac.uk \\ ${ }^{2}$ Observatoire de Paris-Meudon, LESIA, France \\ email: Lidia.vanDriel@obspm.fr \\ ${ }^{3}$ Konkoly Observatory, Budapest, Hungary
}

\begin{abstract}
.
An overview is presented of large-scale coronal structures as observed in soft X-rays (SXR) and extreme ultraviolet (EUV) wavelengths in the context of their magnetic properties. These structures include large-scale interconnecting and trans-equatorial loops, coronal streamers, coronal holes, filaments and filament channels. Since the general appearance of the corona and its structures change with evolving underlying fields, evolutionary trends and solar cycle dependence of these coronal structures are discussed as well.
\end{abstract}

\section{Introduction}

\subsection{The corona in EUV and SXRs}

When the Sun is viewed in optical wavelengths, the brightness of the photosphere overpowers the brightness of the atmospheric layers. To make the $10^{6}$ times fainter corona visible, one has to cover the bright solar disc (see e.g. Vourlidas, in this issue). Since the solar corona is hot, having million-degree temperatures, when observed in soft Xrays (SXRs) and in the extreme ultraviolet (EUV) wavelength domain, the solar corona appears the brightest while the photosphere becomes invisible, contrary to white-light imaging. The huge advantage of this is that the corona can be seen over the entire solar disk. A disadvantage of SXR and EUV wavelength domains is, however, that these can only be observed from above the terrestrial atmospheric mass, i.e. with space-borne instruments.

\subsection{Our learning curve: historical EUV and SXR observations}

The history of observations of the white-light corona goes back thousands of years (eclipse observations). On the other hand, solar UV, EUV and X-ray observations have been developed only recently, in the last fifty years.

The first observational evidence for solar $U V$ emission (in spectral lines) was found in 1947 by R. Tousey of NRL using captured German V2 rockets. Solar X-rays were first detected by T.R Burnight, using a rocket-borne pinhole camera. The first solar SXR image is shown in Figure 1a. The rocket flights were followed by the Orbiting Solar Observatory series (1-8) in the period 1962-1975, carrying EUV, SXR and hard X-ray (HXR) spectrometers and spectro-heliographs on-board. The manned Skylab observations carried out in 1973-74 with UV and X-ray telescopes represented a leap in our knowledge of the solar corona. A Skylab SXR image is shown in Figure 1b. The next major solar EUV 

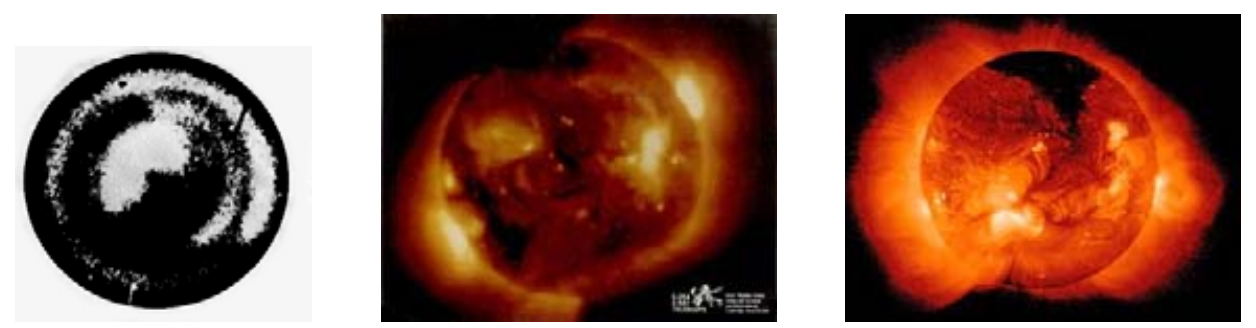

Figure 1. Our evolving view of the SXR Sun. (a): SXR image of the Sun taken with a rocket-borne pinhole camera in 1960; (b): Skylab/S054 image taken in 1973; (c): Yohkoh/SXT image taken in 1992. Note that the brightest features are active regions (ARs), which are gradually resolved into loops. The large dark areas are coronal holes, which are the most extended around the poles, but also appear at low latitures. X-ray bright points are related to small-scale magnetic bipoles.

and SXR space experiments were the greatly successful Solar Maximum Mission (SMM; 1980-1989) and the Japanese Hinotory (1981-82). A cycle-long uninterrupted series of observation (1991-2000) of the soft X-ray corona was provided by the Yohkoh spacecraft, which carried four experiments, providing both imaging and spectral information on the dynamic solar corona from SXRs to $\gamma$-rays. The image in Figure 1c is a Yohkoh/SXT observation.

Presently, three major space-borne experiments are providing EUV and SXR data of the Sun: SOHO was launched at the end of 1995: EIT images the Sun in four different EUV wavelength bands using filters, while CDS and SUMER provide EUV spectral scans. TRACE (launched in 1998) provides UV and EUV (filter) imaging with an unprecedented spatial resolution of 1 ". RHESSI (launched in 2002) allows us to image the Sun in HXRs and $\gamma$-rays.

A more detailed historical overview of the UV to SXR observations of the Sun from its pioneering rocket-borne experiments up to our present experiments is presented by Aschwanden (2004).

\section{Physical conditions in the corona and their consequences}

\subsection{The consequence of high temperature: magnetic coupling}

In the million-degree corona both the hydrogen and helium are fully ionised, while other elements are in a partially ionised state. The highly ionised corona is in a plasma state, which has a special relationship with the magnetic field: field lines move as if they were "frozen into" the plasma (Alfvén, 1943), there is virtually no relative motion between the plasma and the magnetic field perpendicular to the field. However, plasma can flow along the field lines. Furthermore, thermal conduction is anisotropic, mostly parallel to the field lines.

As a consequence of this special relationship coronal loops and coronal holes allow us to visualize the structure of coronal magnetic fields, since many radiation mechanisms are directly coupled to the dissipation of magnetic energy, which results in plasma heating.

\subsection{Magnetic fields in the corona}

Coronal magnetic fields have highly inhomogeneous photospheric boundary conditions ranging from sunspots $(B=2000-4000 \mathrm{G})$ through faculae $(100-300 \mathrm{G}$ with $1100 \mathrm{G}$ concentrations) down to the quiet-sun and coronal hole level (10-50 G, with a very low net value). Note that filling factor is important and the latter $10-50 \mathrm{G}$ fields may contain unresolved $1 \mathrm{kG}$ concentrations. 
Magnetic fields are well measured in the photosphere, while in the corona it is very difficult to measure them. However, there is great progress in this area and coronal magnetic field measurements are becoming increasingly reliable (Lagg, 2006). Measurement methods include the Zeeman effect used in infrared spectral lines, Zeeman and Hanle effects in prominence, loop oscillations, magnetic field extrapolations and several radio methods using e.g. microwave gyro-resonance emission, type II and III bursts. For more details see the paper in this issue by Lagg (2006).

Globally, magnetic fields decrease steeply with height. Combining results derived using many of the above methods Dulk and Mclean (1978) obtained the following magnetic field strength hight relations: $B(R)-0.5\left(\left(R / R_{\odot}\right)-1\right)^{-1.5} \mathrm{G}$ for the height range $1.02 \leqslant$ $R / R_{\odot} \leqslant 10$. However, there are two orders of magnitude deviations from this relation locally!

\subsection{Role of the plasma- $\beta$}

The magnitude of the plasma pressure $p$ and magnetic pressure $B^{2} / 2 \mu_{0}$ (where $\mu_{0}$ is the magnetic permeability) is compared in the plasma $\beta$ parameter, which is defined as: $\beta=2 \mu_{0} p / B^{2}$. Below the photosphere $\beta<1$, thus gas pressure dominates. In the corona $\beta>1$, thus magnetic pressure dominates and there is no cross-field transport. However, at about $0.2 \mathrm{R}_{\odot}$ high $\beta$ values may occur, e.g. in the streamer regions having hot plasma and weak magnetic field. In such regions hot plasma can leak out across the cusped magnetic field lines (Gary, 2001). It is noteworthy that in the present calculations of $\beta$ with heigh in the corona, due to the lack of direct magnetic measurements, there is a great dependence on the magnetic model used - B is still the least known observable!

\section{Magnetic structures in the corona}

Coronal magnetic structures include:

- Bipolar bright point loops

- Active region (AR) loops

- AR to AR or trans-equatorial loops (TELs)

- Coronal streamers

- Coronal holes (CHs)

- Filaments and filament channels

Furthermore, the corona is the source of the fast and slow solar winds, and transient events like flares, jets, filament eruptions and coronal mass ejections (CMEs). Transient events can considerably change the structure of the corona, since, especially coronal mass ejections frequently lead to global magnetic re-organisation.

Since this is a review on large-scale coronal structures, I do not discuss bright points and active region loops here.

Starting with the simplest classification, coronal structures appear bright (arcades, loops, helmet streamers) or dark (CHs). The difference in brightness in EUV and SXR wavelengths is mainly due to differences in plasma density $(\rho)$ and temperature $(T)$. Density and temperature greatly depend on the magnetic field topology: bright features have closed magnetic structures (BP and AR loops), while dark CHs have an "open" magnetic structure towards the interplanetary magnetic field.

\subsection{Trans-equatorial loops}

\subsubsection{General properties}

Long loops, that connect ARs on opposite sides of the equator were discovered in EUV and SXRs in Skylab data and referred to as "trans-equatorial loops" or TELs (Sheeley et al., 1975; Chase et al., 1976; Svestka et al., 1977; Sakurai and Uchida, 1977). 
TELs play an important role in Babcock's dynamo model in the restoration of the poloidal field. Furthermore, TELs can provide clues for the behaviour of the dynamo (Sakurai and Uchida, 1977). Emerging and reconnected TELS play an important part even in modern dynamo models (see Dikpati, 2006, in this issue).

TELs provide us with a link to understand the global reconstruction of large-scale magnetic fields that must take place following a CME (Wang et al., 2001). Since TELs are magnetic connections between different ARs, they may channel energy between their distant footpoint ARs and can be linked to sympathetic flaring (e.g. Moon et al., 2002).

Pevtsov (2000) made a systematic study of 87 TELs using seven years of Yohkoh/SXT observations. His main results include:

bullet One-third of ARs exibit TELs, and this fraction is cycle invariant. An addition to this result: Chase et al. (1976) found 100 coronal loops connecting 94 AR, 20 of them TELs. Therefore, TELs are not rare!

bullet In the sample, $60 \%$ were so-called "connection type", while $30 \%$ showed signatures of being recently formed by reconnection. Corresponding to their shape, they were put in the X or Y "reconnection type" category.

bullet The length of TELs was found to range from 100 to $900 \mathrm{Mm}$, corresponding to 10-75 heliographic degrees). The mean length was $300 \mathrm{Mm}\left(30^{\circ}\right)$.

bullet TELs foot-points rotate with the same rate $\left(87 \%\right.$ differ with less than $1^{\circ}$ day $\left.^{-1}\right)$, and the same handedness of the magnetic field (of more than $68 \%$ ).

Chen et al., (2006), using the same data source as Pevtsov (2000), but classifying TELs according to magnetic polarities of the footpoints, showed that about $2 / 3$ of all TELs connect leading magnetic polarities, leaving $1 / 3$ to connect following magnetic polarities of ARs. They found this rule independent of the solar cycle (after having analysed two half-cycles covered by Yohkoh observations). They found (a plausible) dependence of the length of TELs on the solar cycle: at the start of the cycle, when the heliographic latitude of ARs is high, characteristically large foot-point separation was found, while at the end of the cycle, when ARs were closer to the equator, foot-point separation (length) of TELs decreased. Adding to Pevtsov's (2000) results on the similar rotation rate of TELs' foot-points, they showed that TEL foot-points are symmetric in latitude.

\subsubsection{Multi-wavelength properties and activity}

Fárnik et al. (2001), while investigating the characteristics of TELs' foot-points in a limited sample, noted a surprising lack of EUV counterparts of SXR TELs. However, Glower et al. (2003) showed that most of SXR TELs do have EUV counterparts. Brosius (2006), using SOHO/CDS data found multi-temperature properties in a TEL, with temperatures ranging over two orders of magnitude.

Harra et al. (2003) showed evidence for flaring behaviour of a TEL: (i) finding a cusped structure in the hot SXRs, while (ii) evidence for cooling flows in the cooler chromospheric and transition region lines.

TELs are sometimes related to and known to erupt during CMEs (Delannée and Aulanier, 1999; Khan and Hudson, 2000; Wang et al., 2001). Glover et al. (2003) showed in a sample of 18 TELs that the longer the loop, the more likely that it erupts.

\section{Helmet streamers}

Helmet streamers are huge, long-lived, mostly radially oriented structures extending from the base of the corona to several solar radii. Helmet streamers are rooted in elongated bipolar magnetic structures, the lower part consists of an arcade of closed loops, the top 
tapers into a thin, elongated streamer. Below the arcade of loops usually there is a lowdensity cavity, with a prominence in its bottom part. Helmet streamers may get (partly) disrupted, but they re-form in most cases. Since the properties of white-light streamers are discussed in great details by Vourlidas (2006, in this issue), I refer the reader to this article for more details.

In SXRs and EUV, however, helmet streamers appear after an eruption, when the field lines, which had been "opened up" by the eruption start reconnecting. Such a SXR helmet streamer consists of an arcade of hot reconnected loops enveloped by an elongated helmet containing a long current sheet (Forbes and Acton, 1996).

Helmet streamers may also be disturbed by a near-by CME. Fazakerley et al. (2005) argued that a CME observed on 20 January 2004 at 00 UT interacted and reconnected with a neighbouring streamer, leading to magnetic disconnection on one side, corroborated by uni-directional electron flows observed in situ in the related interplanetary magnetic cloud.

\section{Coronal holes}

Coronal holes (CHs; Waldmeier, 1956) are darker areas in the solar corona, characterised by lower density and temperature. They are situated over areas of monopolar magnetic field (which, however, contains a weak magnetic carpet-type mixed-polarity population as well). Their magnetic field lines are "open" towards the interplanetary space. CHs are are the source of fast solar wind streams and recurrent geomagnetic storms (Nolte et al., 1976). CHs are best observed in EUV, SXR and radio wavelengths, but they are also noted in limb observations, $\mathrm{HI} \mathrm{D}_{3}$ and $1083 \mathrm{~nm}$ lines.

Following Harvey (1996), CHs can be classified as:

- polar

- non-polar (isolated)

- transient (associated with CMEs)

Non-polar (isolated) CHs can be (i) AR-related and (ii) background-field related. The former $\mathrm{CHs}$ show differential rotation similarly to ARs, while the latter show quasi-rigid rotation.

\subsection{Polar $\mathrm{CHs}$}

Polar CHs evolve from mid- and high-latitude $\left(>60^{\circ}\right.$ ) isolated CHs (Webb et al., 1984; Fox et al., 1998; Harvey and Recely, 2002). Such isolated CHs form in the follower magnetic polarity areas of old bipolar regions just before the completion of the polar polarity reversal during solar maximum. These $\mathrm{CHs}$ expand to cover the poles within three solar rotations (!) after the reversal of the pole. The forming polar $\mathrm{CH}$ is asymmetric at first (Harvey and Recely, 2002). During the initial 1.2-1.4 years polar CHs have lobes extending to active longitudes. After that the $\mathrm{CH}$ area and magnetic flux first fluctuates, then shows a gradual increase finally reaching maximum during cycle minimum.

Polar CHs are largest at cycle minimum, covering $\approx 15 \%$ of the Sun (Sheeley, 1980). Then their magnetic flux is about $3 \times 10^{22} \mathrm{Mx}$, representing about $30 \%$ of the total solar flux, with a flux density of $7-8 \mathrm{Mx} \mathrm{cm}^{-2}$ (Harvey and Recely, 2002). Polar CHs decrease both in area and flux as the new cycle evolves and disappear completely 1.1-1.8 years before polarity reversal. The shrinking polar CHs are asymmetric (Broussard et al., 1978; Waldmeier, 1981; Harvey and Recely, 2002). Polar CHs disappear, when their magnetic flux becomes less than $7-9 \times 10^{21} \mathrm{Mx}$ above $70^{\circ}$ latitude (Harvey and Recely, 2002). 
The lifetime of polar CHs, based on the above history, is 7-9 years (Waldmeier, 1981; Harvey and Recely, 2002).

\subsection{Non-polar (isolated) CHs}

Magnetic polarities are greatly unbalanced in isolated CHs: above $20 \mathrm{G} 95 \%$ of the field represents the dominant polarity (Zhang, 2006, in this issue). However, how do these polarities relate to the polarity of the polar $\mathrm{CH}$ at any given time? Extensions of polar coronal holes have the same polarity all over a given hemisphere, identical to the polarity of the polar CH. Non-polar (isolated) CHs are unipolar, but both polarities are found on both hemispheres at cycle maximum, with clusters of the same polarity along certain longitudes (Bilenko, 2004). Both the total number and area of isolated CHs increase with the cycle from minimum to maximum (McIntosh et al., 1992; Bilenko, 2002). AR nests are related to mixed polarity $\mathrm{CH}$ clusters, the more complex the nest, the larger are the $\mathrm{CH}$ areas (Bilenko, 2004).

\subsection{Rigid rotation of $\mathrm{CHs}$}

The famous Skylab observations of a trans-equatorial coronal hole between 1 June 14 October 1973 revealed a puzzling fact: coronal holes rotate quasi-rigidly, while the underlying photosphere in which their open field lines are rooted, rotate differentially. Such paradox can be solved supposing that continuous magnetic (inter-change, Crooker et al., 2002) reconnection is taking place around the $\mathrm{CH}$ boundaries, which counteract the rotational shearing of the holes via continual sideways displacements (e.g. Nash et al., 1988; Wang and Sheeley, 2004).

First observational evidences for the presence of small-scale, low-energy reconnection events along $\mathrm{CH}$ boundaries were presented by Madjarska et al. (2004), who found a higher number-density of bi-directional jets (in SOHO/SUMER data) along an equatorial $\mathrm{CH}$ boundary than elsewhere. Comparison of quiet-sun and $\mathrm{CH}$ boundary intensities by Raju et al. (2005) supported this scenario.

\subsection{CH affected by CME activity}

Attrill et al. (2006) analysed the effect of a CME on a polar CH: they provided multiwavelength evidence to show that the expanding magnetic structure of the CME on 12 May 1997 reconnected with the open magnetic field lines of the northern polar $\mathrm{CH}$, leading to the formation of bright SXT loops between the coronal dimming and $\mathrm{CH}$ regions and a retraction of the $\mathrm{CH}$ boundary (interchange reconnection). Such interaction has been seen before: Hudson et al. (1996) called it the "aurora solaris" phenomenon. Sterling and Moore (2001) "crinkles" have a similar origin.

\section{Filaments and filament channels}

Filaments (or prominences when seen on the limb) and the corridor surrounding them are characteristic large-scale features in a transition region or coronal image. Filaments are higher density $\left(\rho \approx 10^{9}-10^{11} \mathrm{~cm}^{-3}\right)$, lower temperature $\left(\mathrm{T} \approx 10^{4}\right)$ regions than the surrounding corona. They lie along magnetic inversion lines. Largescale magnetic structures are, in general, close to potential. However, this is not true for filaments! Filaments and filament channels are highly sheared magnetic structures. There are two major groups: (i) AR filaments and (ii) quiescent filaments. The former are lower-lying denser formationsthan the latter ones. Filaments (especially in their 
quiescent stage) may live for several months, during which time they erupt and re-form several times.

What kind of mechanism can allow cool and dense matter to be supported and maintained in the hot and tenuous environment of the corona? The answer is: magnetic fields! Models for the magnetic support of a prominence generally consider that the cool and dense material is suspended in dips of magnetic field lines. Milestone models were proposed by:

(i) Kippenhahn and Schlüter (1957), who suggested that magnetic tension provides the balancing force;

(ii) Kuperus and Raadu (1974) predicted an inverse magnetic field direction (i.e. opposite to the direction of the magnetic arcade of the filament channel) found observationally in prominences;

(iii) Aulanier and Démoulin (1998) proposed that prominence material is supported in the dips of a large, mildly twisted flux tube. These authors, using linear force-free and magneto-hydrostatic extrapolations (LMHS method), could actually reproduce the morphology of observed filaments, including their feet or barb structures (Aulanier et al, 1999, 2000).

Providing observational support to the magnetic dip models of prominences, López Ariste et al. (2006) has indeed found a concave-up magnetic topology in a filament channel using vector magnetic measurements in the $\mathrm{H} \alpha$, NaD1 and FeI lines.

In EUV, the typically observed structure of a filament is a wide region located along the filament axis, which corresponds to an intensity depletion. This region is usually referred to as the "EUV filament channel" (EFC), which was first noted in Skylab and OSO observations (Orrall and Schmall, 1976, 1979). The width of an EFC can vary from 10 to $50 \mathrm{Mm}$ along its axis, which is on average five times wider than its related $\mathrm{H} \alpha$ filament body. Aulanier and Schmieder (2002), using the LMHS method, found good correspondence between the EUV filament channel and the distribution of calculated magnetic dips, which are formed at low altitude above dispersed photospheric parasitic polarities. In these magnetic dips plasma condensations form, which absorb background EUV radiation. Towards the centre of the channel the dips become less shallow, providing a smooth transition to $\mathrm{H} \alpha$ feet features and the filament body itself.

For more details on filaments I refer the reader to the paper by Schmieder (2006, in this issue).

\section{Large-scale coronal magnetic fields and MHD simulations of coronal structure}

The large-scale coronal structures observed are produced by the interaction of the solar wind with the coronal magnetic field. Advances in coronal modelling techniques now allow realistic simulations of this interaction based on the MHD equations. The comparison between coronal models and observations is very useful to show us the level of understanding reached in this field, and it is especially challenging, when it appears in the form of a forecast.

Mikic et al. (1999, 2000) developed a method to model coronal field and WL and EUV radiation of plasma contained in coronal magnetic structures. They already used their method for forecasting the shape of the corona during the 11 August 1999 solar eclipse. Now they used their improved method to do the same for the 29 March 2006 eclipse, which occurred just before IAU Symposium 233 and was observed by many of its participants. 

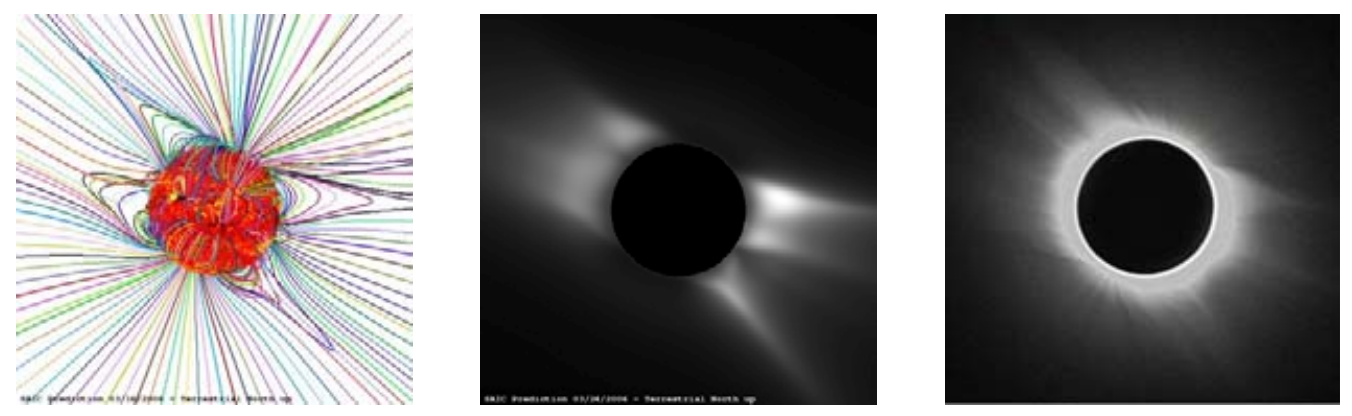

Figure 2. Predicted and observed shape of the eclipse corona on 29 March 2006. Left and middle: MHD simulations by Mikic, Linker et al. (2006). The middle image has been radially detrended to account for the fall-off of the coronal polarisation brightness with height. Right: photograph taken during the eclipse (courtesy Wim van Driel).

For the coronal magnetic field model boundary condition on the radial magnetic field $\mathrm{B}_{r}$ a series of $\mathrm{SOHO} / \mathrm{MDI}$ synoptic magnetic maps were used. Since magnetic fields evolve between the last available synoptic map and the epoch of the eclipse, a forecasted synoptic magnetic pattern was provided by K. Schrijver and M. DeRosa (for details of the method, see Schrijver and DeRosa, 2003). The photospheric field and uniform assumed density $(\rho)$ and temperature $(\mathrm{T})$ at the photosphere were used to solve the MHD equations to steady state in the corona, expressing interaction with the solar wind. The energy equation included the effects of coronal heating, heat conduction, radiative losses, and effects of Alfvén waves plasma $\mathrm{T}$ and $\rho$. Emission of radiation from the corona was computed by integrating the emission kernels for EUV and X-ray radiation along a chosen line of sight. This integration requires knowledge of the plasma density and temperature in the corona, which was predicted by the model (Mikic, Linker, et al., 2006). Surface magnetic fields and polarisation brightness (pB) were obtained from these 3-D MHD simulations by making a line-of-sight integral of the product of the electron $\rho$ and a scattering function. The pB signal is produced by white light scattered off electrons in the coronal plasma and thus (after compensation for the rapid fall off of the coronal density with radial distance) can be used to simulate an eclipse image (Figure 2). Comparison with the actual eclipse photograph shows a good match: a success!

\section{Concluding remarks}

Large-scale coronal structures are defined by the magnetic fields. Since solar magnetic fields are dynamic, coronal structures show changes on all time-scales from a few minutes to the length of the cycle. As the successful forecast of the 29 March 2006 eclipse corona (Mikic, Linker et al., 2006) evidences, our understanding of large-scale coronal structures is improving. Future space missions, Solar-B, Stereo and the Solar Dynamic Observatory will open up unprecedented paths towards an even deeper understanding of the physical nature of the solar magnetic field and it's influence on the coronal plasma.

\section{Acknowledgements}

I acknowledge the Hungarian government grant OTKA 048961 and Guillaume Aulanier for a critical reading of the manuscript. 


\section{References}

Alfvén, H. 1943, Srk. F. Mat. Ast. Fys. 29A, 1

Aschwanden, M.J. 2004, Physics of the Solar Corona. An Introduction. Praxis Publishing LTd., Chichester, UK and Springer-Verlah, Berlin

Attrill, G., Nakwacki, M.S., Harra, L.K., van Driel-Gesztelyi, L., Mandrini, C.H., Dasso, S., and Wang, J. 2006, Solar Phys. in press

Aulanier G., Démoulin P. 1998, A\&A 329, 1125

Aulanier, G., Démoulin, P., Mein, N., et al. 1999, A\&A, 432, 867

Aulanier, G., Srivastava, N., Martin, S.F. 2000, ApJ 543, 447

Aulanier G., Schmieder B. 2002, A\&A 386, 1106

Bilenko, I. A. 2004, Solar Phys. 221, 261

Broussard, R.M., Sheeley, N.R. Jr., Tousey, R., Underwood, J.H. 1978, Solar Phys. 56, 161

Chase, R.C., Krieger, A.S., Švestka, Z., Vaiana, G.S. 1976, in Space Research XVI, (Berlin: Akademie), 917

Chen, J., Bao, S., Zhang, H. 2006, Solar Phys. 235, 281

Crooker, N.U., Gosling, J.T., Kahler, S.W. 2002, JGR, 107, A2, 1028

Delannée, C., Aulanier, G. 1999, Solar Phys. 190, 107

Dikpati, M. 2006, in this issue

Dulk, G.A., McLean, D.J. 1978, Solar Phys. 57, 279

Fárník, F., Karlický, M., Švestka, Z. 2001, Solar Phys. 202, 81

Fazakerley, A.N., Harra, L.K., Culhane, J.L., van Driel-Gesztelyi, L., Lucek, E., Matthews, S.A., Owen, C.J., Mazelle, C., Balogh, A., and Réme, H. 2005, Geophys. Res. Let. 32, 13, L13105

Forbes, T.G., Acton, L.W. 1996, ApJ 459, 330

Fox, P. McIntosh, P. Wilson, P.R. 1998, Solar Phys. 177, 375

Gary, G.A. 2001, Solar Phys. 203, 71

Glover, A., Harra, L.K., Matthews, S.A., Foley, C.A. 2003, A\&A 400, 759

Harra, L.K., Matthews, S.A., van Driel-Gesztelyi, L. 2003, ApJ 598, L59

Harvey, K.L. 1996, in Proc. Eighth Solar Wind Conf. AIP Conf. Proc. 382, 9

Harvey, K.L., Recely, F. 2002, Solar Phys. 211, 31

Hudson, H.S., Acton, L.W., and Freeland, S.L. ApJ 470, 629

Kippenhahn R., Schlüter A. 1957, Zs. Ap. 43, 36

Khan and Hudson, 2000, Geophys. Res. Let. 27, 8, 183

Kuperus M., Raadu M.A. 1974, A\&A 31, 189

Lagg, A, 2006, in this issue

López Ariste, A., Aulanier, G., Schmieder, B., Sainz Dalda, A. 2006, A\&A in press

Madjarska, M.S., Doyle, J.G., van Driel-Gesztelyi, L. 2004, ApJ 603, L57

Mikic, Z., Linker, J.A., Schnack, D.D., Lionello, R., Tarditi, A., 1999, Phys. Plasmas, 6, 2217

Mikic, Z., Linker, J.A., Riley, P., Lionello, R., 2000, ASP Conf. Ser. 205, p. 162

Mikic, Z., Linker, J.A., et al. 2006, http://imhd.net

McIntosh, P.S., Thompson, R.j., Willock, E.C. 1992, Nature, 360, 322

Moon, Y.-J., Choe, G.S., Park, Y.D., Wang, H., Gallagher, P.T., Chae, J., Yun, H.S., Goode, P. 2002, ApJ 574, 434

Nash, A.G., Sheeley, N.R., Jr., Wang, Y.-M. 1988, Solar Phys., 117, 359

Nolte, J.T., Krieger, A.S., Timothy, A.F., Gold, R.E., Roelof, E.C., Vaiana, G., Lazarus, A.J., Sullivan, J.D., McIntosh, P.S. 1978, Solar Phys. 46, 303

Orrall, E.Q., Schmahl, E.J. 1976, Solar Phys., 50, 365

Orrall, E.Q., Schmahl, E.J. 1979, ApJ, 231, L41

Pevtsov. A.A. 2000, ApJ 531, 553

Raju, K.P., Bromage, B.J.I., Chapman, S.A., Del Zanna, G. 2005, A\&A 432, 341

Sakurai, T., Uchida, Y. 1977, Solar Phys. 52, 397

Schmieder, B. 2006, in this issue

Schrijver, C.J., DeRosa, M.L. 2003, Solar Phys. 212, 165

Sheeley, N.R.Jr., Bohlin, J.D., Brückner, G.E., Purcell, J.D., Scherrer, V.,Tousey, R. 1975, Solar Phys. 40, 103 
Sterling, A.C., Moore, R.L. 2001, ApJ 560, 1045

Švestka, Z., Krieger, A.S., Chase, R.C., Howard, R. 1977, Solar Phys. 52, 69

Waldmeier, M. 1957, Die Sonnencorona, Vol. II, Birchauser, Basel, p. 65

Waldmeier, M. 1981, Solar Phys. 70, 251

Wang, H., Chae, J., Yurchyshyn, V., Yang, G., Steinegger, M., Goode, P. 2001, ApJ 559, 1171

Wang, Y.-M., Sheeley, N.R. Jr. 2004, ApJ 612, 1196

Webb, D.F., Davis, J.M., McIntosh, P.S. 1984, Solar Physics, 92, 109

Vourlidas, A. 2006, in this issue

Zhang, J. 2006, in this issue 